\title{
CORRELATED FRACTIONAL COUNTING PROCESSES ON A FINITE-TIME INTERVAL
}

\author{
LUISA BEGHIN* AND \\ ROBERTO GARRA,** Sapienza University of Rome \\ CLAUDIO MACCI,** University of Rome Tor Vergata
}

\begin{abstract}
We present some correlated fractional counting processes on a finite-time interval. This will be done by considering a slight generalization of the processes in Borges et al. (2012). The main case concerns a class of space-time fractional Poisson processes and, when the correlation parameter is equal to 0 , the univariate distributions coincide with those of the space-time fractional Poisson process in Orsingher and Polito (2012). On the one hand, when we consider the time fractional Poisson process, the multivariate finitedimensional distributions are different from those presented for the renewal process in Politi et al. (2011). We also consider a case concerning a class of fractional negative binomial processes.
\end{abstract}

Keywords: Poisson process; negative binomial process; weighted process

2010 Mathematics Subject Classification: Primary 33E12; 60G22; 60G55

Secondary 60E05

\section{Introduction}

Several fractional processes in the literature are defined by considering some known equations in terms of suitable fractional derivatives. In this paper we are interested in particular Lévy counting processes, as in the recent paper [6]; in particular, we deal with Poisson and negative binomial processes. There is an extensive literature on fractional Poisson processes; see, e.g. [7], [8], [16], [19], [24], and [26] (we also cite [15] and [20] where their representation in terms of randomly time-changed and subordinated processes was studied in detail). For fractional negative binomial processes; see, e.g. [6, Example 3] and [28]. Among the other fractional processes studied in the literature, we recall the diffusive processes [2], [3], [18], [22], [27], the telegraph processes [21], and the pure-birth processes [23].

Often the results for these fractional processes are given in terms of the Mittag-Leffler function

$$
E_{\alpha, \beta}(x):=\sum_{r \geq 0} \frac{x^{r}}{\Gamma(\alpha r+\beta)}
$$

\footnotetext{
Received 25 July 2014; revision received 26 September 2014.

* Postal address: Dipartimento di Scienze Statistiche, Sapienza Università di Roma, Piazzale Aldo Moro 5, I-00185 Roma, Italy. Email address: luisa.beghin@uniroma1.it

** Postal address: Dipartimento di Scienze di Base e Applicate per l'Ingegneria, Sapienza Università di Roma, Via A. Scarpa 16, I-00161 Roma, Italy. Email address: rolinipame@yahoo.it

*** Postal address: Dipartimento di Matematica, Università di Roma Tor Vergata, Via della Ricerca Scientifica, I-00133

Rome, Italy. Email address: macci@mat.uniroma2.it
} 
see, e.g. [25, p. 17]. We also recall the generalized Mittag-Leffler function

$$
E_{\alpha, \beta}^{\gamma}(x):=\sum_{r \geq 0} \frac{(\gamma)^{(r)} x^{r}}{r ! \Gamma(\alpha r+\beta)}
$$

where, for $\gamma \in \mathbb{R}$,

$$
(\gamma)^{(r)}:= \begin{cases}\gamma(\gamma+1) \cdots(\gamma+r-1) & \text { if } r \geq 1 \\ 1 & \text { if } r=0\end{cases}
$$

is the rising factorial (also called the Pochhammer symbol), and $E_{\alpha, \beta}^{\gamma}$ coincides with $E_{\alpha, \beta}$ when $\gamma=1$.

In this paper we consider some processes $\left\{N_{\rho}(\cdot): \rho \in[0,1]\right\}$ on a finite-time interval $[0, T]$ for some $T \in(0, \infty)$. More precisely, $N_{\rho}(\cdot)=\left\{N_{\rho}(t): t \in[0, T]\right\}$ is defined by

$$
N_{\rho}(t):=\sum_{n=1}^{M_{g}} \mathbf{1}_{[0, t]}\left(X_{n}^{F, \rho}\right)
$$

where $M_{g}$ is a nonnegative integer-valued random variable with probability generating function (PGF) $g$, i.e. $g(u):=\mathbb{E}\left[u^{M_{g}}\right]$, and $\left\{X_{n}^{F, \rho}: n \geq 1\right\}$ is a sequence of random variables with (common) distribution function $F$ such that $F(0)=0$ and $F(T)=1$, and independent of $M_{g}$; moreover, the correlation coefficient between any pair of random variables $X_{n}$ and $X_{m}$, with $n \neq m$, is equal to a common value $\rho \in[0,1]$.

Remark 1.1. We have $N_{\rho}(T)=M_{g}$; thus, the distribution of $N_{\rho}(T)$ does not depend on $\rho$.

In this way we are considering a slight generalization of the processes presented in [9]; indeed, we can recover several formulas in [9] by setting $g(u)=\mathrm{e}^{\lambda(u-1)}$ for some $\lambda>0$ (which concerns a Poisson distributed random variable with mean $\lambda$ ), and $F(t)=t$ for $t \in[0,1]$, where $T=1$. The case without correlation, i.e. the $\rho=0$ case, appears in [4]; see also [17], where that process is considered as a claim number process in insurance. Here, in view of what follows, we recall the following formulas; see, e.g. [9, Equations (9) and (10)]). We have the PGF

$$
G_{N_{\rho}(t)}(u)=\rho(1-F(t))+\rho F(t) g(u)+(1-\rho) g(1-F(t)+F(t) u),
$$

and the probability mass function (PMF)

$$
\begin{aligned}
\mathbb{P}\left(N_{\rho}(t)=k\right)= & (1-\rho) \mathbb{P}\left(N_{0}(t)=k\right) \\
& +\rho\left\{(1-F(t)) \mathbf{1}_{\{k=0\}}+F(t) \mathbb{P}\left(M_{g}=k\right)\right\} \quad \text { for all } k \geq 0,
\end{aligned}
$$

where

$$
\mathbb{P}\left(N_{0}(t)=k\right)=\sum_{n=k}^{\infty}\left(\begin{array}{l}
n \\
k
\end{array}\right) F^{k}(t)(1-F(t))^{n-k} \mathbb{P}\left(M_{g}=n\right) \quad \text { for all } k \geq 0
$$

concerns the $\rho=0$ case; see [4, Equation (2.4)].

As pointed out in [4], this class of counting processes can be useful to tackle the problem of overdispersion and underdispersion in the analysis of count data where correlations between events are present. A possible application can be given, for example, in models 
of nonexponential extinction of radiation in correlated random media; see, e.g. [13]. We also remark that, as far as the marginal distribution of each random variable $N_{\rho}(t)$, in (1.2) we have a mixture between three PMFs, i.e. $\left\{\mathbb{P}\left(N_{0}=k\right): k \geq 0\right\},\left\{\mathbf{1}_{\{k=0\}}: k \geq 0\right\}$, and $\left\{\mathbb{P}\left(M_{g}=k\right): k \geq 0\right\}$, and the weights are $1-\rho, \rho(1-F(t))$, and $\rho F(t)$, respectively.

The aim of this paper is to present some correlated fractional counting processes by choosing, in a suitable way, the PGF $g$ and a distribution function $F$ above. In Section 2 we present a class of space-time fractional Poisson processes (in fact we have the same univariate distributions of the space-time fractional Poisson process in [24] when $\rho=0$ ). A class of fractional negative binomial processes is presented in Section 3.

Finally, since the presentation of the results in [9] refers to the concept of weighted Poisson processes (see also [4] concerning the $\rho=0$ case), in Section 4 we give some minor results on weighted processes. Even though this section seems to be disconnected from the other sections in this paper, in our opinion it is a nice enrichment of the content of [9].

\section{A class of correlated fractional Poisson processes}

For the aims of this section, some preliminaries are needed. First, we consider the Caputo (left fractional) derivative $\mathrm{d}^{\nu} / \mathrm{d} t^{\nu}$ of order $v>0$ (see, e.g. ${ }^{C} D_{a+}^{v}$ in [12, Equations (2.4.14) and (2.4.15)] with $a=0$. We use the notation $[x]:=\max \{k \in \mathbb{Z}: k \leq x\}$ ) defined by, for all $t \geq 0$

$$
\frac{\mathrm{d}^{\nu}}{\mathrm{d} t^{\nu}} f(t):= \begin{cases}\frac{1}{\Gamma(n-\nu)} \int_{0}^{t} \frac{1}{(t-s)^{\nu-n+1}} \frac{\mathrm{d}^{n}}{\mathrm{~d} s^{n}} f(s) \mathrm{d} s & \\ (\text { where } n=[\nu]+1), & \text { if } v \text { is not integer, } \\ \frac{\mathrm{d}^{\nu}}{\mathrm{d} t^{\nu}} f(t) & \text { if } \nu \text { is integer. }\end{cases}
$$

Note that, since here we consider $v \in(0,1]$, we have (see, e.g. [12, Equation (2.4.17)] with $a=0$ ), for all $t \geq 0$,

$$
\frac{\mathrm{d}^{\nu}}{\mathrm{d} t^{\nu}} f(t):= \begin{cases}\frac{1}{\Gamma(1-\nu)} \int_{0}^{t} \frac{1}{(t-s)^{\nu}} \frac{\mathrm{d}}{\mathrm{d} s} f(s) \mathrm{d} s & \text { if } v \in(0,1), \\ \frac{\mathrm{d}}{\mathrm{d} t} f(t) & \text { if } \nu=1 .\end{cases}
$$

We also consider the (fractional) difference operator $(I-B)^{\alpha}$ in [24]. More precisely, $I$ is the identity operator, $B$ is the backward shift operator defined by $B f(k)=f(k-1)$, and $B^{r-1} B f(k)=f(k-r)$ and, therefore,

$$
(I-B)^{\alpha}=\sum_{j=0}^{\infty}(-1)^{j}\left(\begin{array}{c}
\alpha \\
j
\end{array}\right) B^{j} .
$$

We now recall that Orsingher and Polito [24] considered the space-time fractional Poisson process $\left\{N_{0}^{\alpha, v}(t): t \geq 0\right\}$ for $\alpha, v \in(0,1]$, whose PMFs $\left\{p_{k}(t): k \geq 0\right\}$ solve the Cauchy problem:

$$
\frac{\mathrm{d}^{\nu}}{\mathrm{d} t^{\nu}} p_{k}(t)=-\lambda^{\alpha}(I-B)^{\alpha} p_{k}(t), \quad p_{k}(0)= \begin{cases}0, & k>0, \\ 1, & k=0 .\end{cases}
$$

The explicit form of the PGF of this process has the following form (see [24, Equation (2.28)]):

$$
\mathbb{E}\left[u^{N_{0}^{\alpha, v}(t)}\right]=E_{v, 1}\left(-\lambda^{\alpha} t^{\nu}(1-u)^{\alpha}\right) .
$$


In this section we consider a class of correlated space-time fractional Poisson processes on a finite-time interval $[0, T]$. For $\alpha, v \in(0,1]$, we consider $N_{\rho}(\cdot)=N_{\rho}^{\alpha, v}(\cdot)$ such that the PGF of $M_{g}$ is

$$
g(u):=E_{v, 1}\left(-\lambda^{\alpha} T^{\nu}(1-u)^{\alpha}\right),
$$

and the distribution function of the random variables $\left\{X_{n}^{F, \rho}: n \geq 1\right\}$ is

$$
F(t):=\left(\frac{t}{T}\right)^{\nu / \alpha} \quad \text { for } t \in[0, T] .
$$

In what follows we present the PGFs in Proposition 2.1 and the corresponding PMFs in Proposition 2.2. Moreover, in Proposition 2.3, we give an equation for the PMFs in Proposition 2.2 with respect to time $t$.

Proposition 2.1. The PGFs $\left\{G_{N_{\rho}^{\alpha, \nu}(t)}: t \in[0, T]\right\}$ are

$$
\begin{aligned}
G_{N_{\rho}^{\alpha, \nu}(t)}(u)= & \rho\left(1-\left(\frac{t}{T}\right)^{\nu / \alpha}\right)+\rho\left(\frac{t}{T}\right)^{\nu / \alpha} E_{\nu, 1}\left(-\lambda^{\alpha} T^{\nu}(1-u)^{\alpha}\right) \\
& +(1-\rho) E_{\nu, 1}\left(-\lambda^{\alpha} t^{\nu}(1-u)^{\alpha}\right) .
\end{aligned}
$$

Proof. We have

$$
\begin{aligned}
G_{N_{\rho}^{\alpha, \nu}(t)}(u)= & \rho\left(1-\left(\frac{t}{T}\right)^{\nu / \alpha}\right)+\rho\left(\frac{t}{T}\right)^{\nu / \alpha} E_{\nu, 1}\left(-\lambda^{\alpha} T^{\nu}(1-u)^{\alpha}\right) \\
& +(1-\rho) E_{\nu, 1}\left(-\lambda^{\alpha} T^{\nu}\left(1-\left\{1-\left(\frac{t}{T}\right)^{\nu / \alpha}+\left(\frac{t}{T}\right)^{\nu / \alpha} u\right\}\right)^{\alpha}\right)
\end{aligned}
$$

by (1.1), and we conclude with some manipulations of the last term.

Remark 2.1. By Proposition 2.1, if $\rho=0$, we have the PGF

$$
G_{N_{0}^{\alpha, \nu}(t)}(u)=E_{\nu, 1}\left(-\lambda^{\alpha} t^{\nu}(1-u)^{\alpha}\right),
$$

which coincides with the PGF presented in the last case of [24, Table 1]. Note that (2.3) is a generalization of (2.2) with $t \in[0, T]$ instead of $t=T$. Thus, the univariate distributions of the random variables $\left\{N_{0}^{1, \nu}(t): t \in[0, T]\right\}$ (for the $\alpha=1$ case) coincide with the distributions of the random variables of the renewal process $\{M(t): t \in[0, T]\}$ in [26] (restricted to the same finite-time interval). On the other hand, one can check that the multivariate finite-dimensional marginal distributions are different from those in [26] (and, in fact, $\left\{N_{\rho}^{\alpha, \nu}(t): t \in[0, T]\right\}$ is not a renewal process). We explain this with a simple example where we take into account that

$$
\mathbb{P}(M(s)=1)=\mathbb{P}\left(N_{0}^{1, \nu}(s)=1\right)=\lambda s^{\nu} E_{\nu, \nu+1}^{2}\left(-\lambda s^{\nu}\right) \quad \text { for } s \in[0, T]
$$

by [8, Equation (2.5)]. In fact, for $t \in(0, T)$, we have

$$
\mathbb{P}(M(t)=1, M(T)=1)=\lambda t^{\nu} E_{v, v+1}^{2}\left(-\lambda t^{\nu}\right) E_{\nu, 1}\left(-\lambda(T-t)^{\nu}\right)
$$

by combining [26, Equations (11) and (14)] (with $\left(t_{1}, t_{2}\right)=(t, T)$ and $\left(n_{1}, n_{2}\right)=(1,1)$ ) with [26, Equations (2) and (4)], and

$$
\mathbb{P}\left(N_{0}^{1, \nu}(t)=1, N_{0}^{1, \nu}(T)=1\right)=\frac{t}{T} \lambda T^{\nu} E_{v, v+1}^{2}\left(-\lambda T^{\nu}\right)
$$

because $\mathbb{P}\left(N_{0}^{\alpha, \nu}(t)=1 \mid N_{0}^{\alpha, \nu}(T)=1\right)=t / T$ by construction. Then (2.4) and (2.5) coincide only for the nonfractional $v=1$ case; see Figure 1 . 


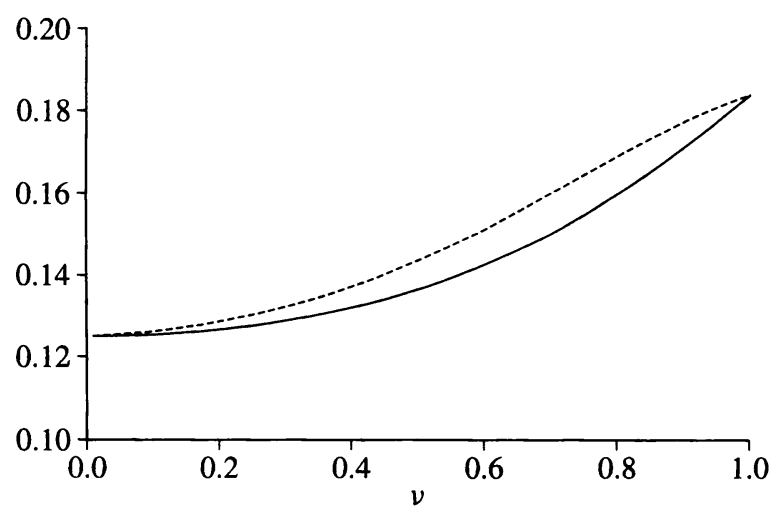

Figure 1: The probabilities (2.4) (dashed line) and (2.5) (solid line) versus $\nu \in(0,1]$ for $t=\frac{1}{2}$ and $T=\lambda=1$.

Proposition 2.2. The PMFs $\left\{\mathbb{P}\left(N_{\rho}^{\alpha, v}(t)=\cdot\right): t \in[0, T]\right\}$ are

$$
\begin{aligned}
& \mathbb{P}\left(N_{\rho}^{\alpha, \nu}(t)=k\right)=(1-\rho) \frac{(-1)^{k}}{k !} \sum_{r=0}^{\infty} \frac{\left(-\lambda^{\alpha} t^{\nu}\right)^{r}}{\Gamma(\nu r+1)} \frac{\Gamma(\alpha r+1)}{\Gamma(\alpha r+1-k)} \\
&+ \rho\left(1-\left(\frac{t}{T}\right)^{\nu / \alpha}\right) \mathbf{1}_{\{k=0\}} \\
&\left.+\left(\frac{t}{T}\right)^{\nu / \alpha} \frac{(-1)^{k}}{k !} \sum_{r=0}^{\infty} \frac{\left(-\lambda^{\alpha} T^{\nu}\right)^{r}}{\Gamma(\nu r+1)} \frac{\Gamma(\alpha r+1)}{\Gamma(\alpha r+1-k)}\right\} \text { for all } k \geq 0
\end{aligned}
$$

Proof. First, we have

$$
\begin{aligned}
\mathbb{P}\left(M_{g}=n\right) & =\mathbb{P}\left(N_{\rho}^{\alpha, \nu}(T)=k\right) \\
& =\frac{(-1)^{n}}{n !} \sum_{r=0}^{\infty} \frac{\left(-\lambda^{\alpha} T^{\nu}\right)^{r}}{\Gamma(\nu r+1)} \frac{\Gamma(\alpha r+1)}{\Gamma(\alpha r+1-n)} \quad \text { for all } n \geq 0
\end{aligned}
$$

by the PGF in (2.2) (see [24, Equation (1.8)]) and by Remark 1.1. Moreover, if we consider (1.3), we obtain

$$
\begin{aligned}
\mathbb{P}\left(N_{0}^{\alpha, \nu}(t)=k\right) \\
=\sum_{n=k}^{\infty}\left(\begin{array}{l}
n \\
k
\end{array}\right)\left(\frac{t}{T}\right)^{(\nu / \alpha) k}\left(1-\left(\frac{t}{T}\right)^{\nu / \alpha}\right)^{n-k} \\
\quad \times \frac{(-1)^{n}}{n !} \sum_{r=0}^{\infty} \frac{\left(-\lambda^{\alpha} T^{\nu}\right)^{r}}{\Gamma(\nu r+1)} \frac{\Gamma(\alpha r+1)}{\Gamma(\alpha r+1-n)} \\
=\frac{(-1)^{k}}{k !}\left(\frac{t}{T}\right)^{(\nu / \alpha) k} \sum_{n=k}^{\infty} \frac{(-1)^{n-k}}{(n-k) !}\left(1-\left(\frac{t}{T}\right)^{\nu / \alpha}\right)^{n-k} \\
\quad \times \sum_{r=0}^{\infty} \frac{\left(-\lambda^{\alpha} t^{\nu}\right)^{r}}{\Gamma(\nu r+1)}\left(\frac{T}{t}\right)^{\nu r} \frac{\Gamma(\alpha r+1)}{\Gamma(\alpha r+1-k)} \frac{\Gamma(\alpha r+1-k)}{\Gamma(\alpha r+1-n)}
\end{aligned}
$$




$$
\begin{aligned}
= & \frac{(-1)^{k}}{k !}\left(\frac{t}{T}\right)^{(\nu / \alpha) k} \sum_{r=0}^{\infty} \frac{\left(-\lambda^{\alpha} t^{\nu}\right)^{r}}{\Gamma(\nu r+1)}\left(\frac{T}{t}\right)^{v r} \frac{\Gamma(\alpha r+1)}{\Gamma(\alpha r+1-k)} \\
& \times \sum_{j=0}^{\infty} \frac{(-1)^{j}}{j !}\left(1-\left(\frac{t}{T}\right)^{\nu / \alpha}\right)^{j} \frac{\Gamma(\alpha r+1-k)}{\Gamma(\alpha r+1-k-j)} \quad \text { for all } k \geq 0 .
\end{aligned}
$$

Then, by the well-known 'Newton's generalized binomial theorem', we obtain

$$
\begin{aligned}
\mathbb{P}\left(N_{0}^{\alpha, \nu}(t)=k\right)= & \frac{(-1)^{k}}{k !}\left(\frac{t}{T}\right)^{(\nu / \alpha) k} \\
& \times \sum_{r=0}^{\infty} \frac{\left(-\lambda^{\alpha} t^{\nu}\right)^{r}}{\Gamma(\nu r+1)}\left(\frac{T}{t}\right)^{v r} \frac{\Gamma(\alpha r+1)}{\Gamma(\alpha r+1-k)}\left(1+\left(\frac{t}{T}\right)^{\nu / \alpha}-1\right)^{\alpha r-k} \\
= & \frac{(-1)^{k}}{k !} \sum_{r=0}^{\infty} \frac{\left(-\lambda^{\alpha} t^{\nu}\right)^{r}}{\Gamma(\nu r+1)}\left(\frac{t}{T}\right)^{(\nu / \alpha) k-v r+v r-(v / \alpha) k} \frac{\Gamma(\alpha r+1)}{\Gamma(\alpha r+1-k)} \\
= & \frac{(-1)^{k}}{k !} \sum_{r=0}^{\infty} \frac{\left(-\lambda^{\alpha} t^{\nu}\right)^{r}}{\Gamma(\nu r+1)} \frac{\Gamma(\alpha r+1)}{\Gamma(\alpha r+1-k)} \quad \text { for all } k \geq 0
\end{aligned}
$$

where, as we expected by (2.3), $\mathbb{P}\left(N_{0}^{\alpha, v}(t)=k\right)$ here meets $\mathbb{P}\left(N_{\rho}^{\alpha, v}(T)=k\right)$ in (2.6) (here we have $t$ and $k$ in place of $T$ and $n$ in (2.6)). We conclude the proof by considering (1.2) together with the last expression above obtained for the $\rho=0$ case.

In view of Proposition 2.3, we remark that in a part of the proof we refer to [5, Theorem 2] which can be derived by referring to a subordinated representation of the space-time fractional Poisson process in terms of both stable subordinator and its inverse; see also [5, Equations (3.20) and (3.1)].

Proposition 2.3. Let $\left\{\mathbb{P}\left(N_{\rho}^{\alpha, \nu}(t)=\cdot\right): t \in[0, T]\right\}$ be the PMFs in Proposition 2.2. Then we have the following equations:

$$
\begin{aligned}
& \frac{\mathrm{d}^{\nu}}{\mathrm{d} t^{\nu}} \mathbb{P}\left(N_{\rho}^{\alpha, \nu}(t)=0\right) \\
& =-\lambda^{\alpha} \mathbb{P}\left(N_{\rho}^{\alpha, v}(t)=0\right)+\lambda^{\alpha} \rho \\
& -\rho\left(\frac{t}{T}\right)^{\nu / \alpha}\left(\lambda^{\alpha}+t^{-v} \frac{\Gamma(\nu / \alpha+1)}{\Gamma(\nu / \alpha-\nu+1)}\right)\left(1-\mathbb{P}\left(N_{\rho}^{\alpha, \nu}(T)=0\right)\right) \quad \text { for } k=0, \\
& \frac{\mathrm{d}^{\nu}}{\mathrm{d} t^{\nu}} \mathbb{P}\left(N_{\rho}^{\alpha, \nu}(t)=k\right) \\
& =-\lambda^{\alpha}(I-B)^{\alpha} \mathbb{P}\left(N_{\rho}^{\alpha, \nu}(t)=k\right)+\lambda^{\alpha} \rho\left(1-\left(\frac{t}{T}\right)^{\nu / \alpha}\right)(-1)^{k}\left(\begin{array}{l}
\alpha \\
k
\end{array}\right) \\
& +\rho\left(\frac{t}{T}\right)^{\nu / \alpha}\left[\lambda^{\alpha}(I-B)^{\alpha}+t^{-\nu} \frac{\Gamma(\nu / \alpha+1)}{\Gamma(\nu / \alpha-\nu+1)}\right] \mathbb{P}\left(N_{\rho}^{\alpha, \nu}(T)=k\right) \quad \text { for all } k \geq 1 \text {. }
\end{aligned}
$$

In all cases we have the initial conditions $\mathbb{P}\left(N_{\rho}^{\alpha, \nu}(0)=0\right)=1$ and $\mathbb{P}\left(N_{\rho}^{\alpha, \nu}(0)=k\right)=0$ for all $k \geq 1$. 
Proof. The initial conditions trivially hold. Throughout this proof we consider the notation

$$
p_{k}^{\alpha, \nu}(t)=\mathbb{P}\left(N_{0}^{\alpha, \nu}(t)=k\right) \text { for all } k \geq 0
$$

for the PMF concerning the $\rho=0$ case. Then, by (1.2) and Remark 1.1, we obtain

$$
\begin{aligned}
\frac{\mathrm{d}^{\nu}}{\mathrm{d} t^{\nu}} \mathbb{P}\left(N_{\rho}^{\alpha, \nu}(t)=k\right)= & (1-\rho) \frac{\mathrm{d}^{\nu}}{\mathrm{d} t^{\nu}} p_{k}^{\alpha, \nu}(t) \\
& +\rho\left\{-\frac{1}{T^{\nu / \alpha}} \mathbf{1}_{\{k=0\}} \frac{\mathrm{d}^{\nu}}{\mathrm{d} t^{\nu}} t^{\nu / \alpha}+\frac{1}{T^{\nu / \alpha}} \mathbb{P}\left(N_{\rho}^{\alpha, \nu}(T)=k\right) \frac{\mathrm{d}^{\nu}}{\mathrm{d} t^{\nu}} t^{\nu / \alpha}\right\} \\
= & (1-\rho) \frac{\mathrm{d}^{\nu}}{\mathrm{d} t^{\nu}} p_{k}^{\alpha, \nu}(t)-\frac{\rho}{T^{\nu / \alpha}}\left\{\mathbf{1}_{\{k=0\}}-\mathbb{P}\left(N_{\rho}^{\alpha, \nu}(T)=k\right)\right\} \frac{\mathrm{d}^{\nu}}{\mathrm{d} t^{\nu}} t^{\nu / \alpha} .
\end{aligned}
$$

Moreover, we have

by [5, Theorem 2] and

$$
\frac{\mathrm{d}^{\nu}}{\mathrm{d} t^{\nu}} p_{k}^{\alpha, \nu}(t)=-\lambda^{\alpha}(I-B)^{\alpha} p_{k}^{\alpha, \nu}(t)
$$

$$
\frac{\mathrm{d}^{\nu}}{\mathrm{d} t^{\nu}} t^{\nu / \alpha}=t^{\nu / \alpha-\nu} \frac{\Gamma(\nu / \alpha+1)}{\Gamma(\nu / \alpha-\nu+1)}
$$

(see, e.g. [12, Equations (2.2.11) and (2.4.8)], or a correction of [12, Equation (2.4.28)]). Then, we obtain

$$
\begin{aligned}
\frac{\mathrm{d}^{\nu}}{\mathrm{d} t^{\nu}} \mathbb{P}\left(N_{\rho}^{\alpha, \nu}(t)=k\right)= & -\lambda^{\alpha}(I-B)^{\alpha}(1-\rho) p_{k}^{\alpha, \nu}(t) \\
& -\rho\left(\frac{t}{T}\right)^{\nu / \alpha}\left\{\mathbf{1}_{\{k=0\}}-\mathbb{P}\left(N_{\rho}^{\alpha, \nu}(T)=k\right)\right\} t^{-v} \frac{\Gamma(\nu / \alpha+1)}{\Gamma(\nu / \alpha-\nu+1)} .
\end{aligned}
$$

From now on we consider the $k=0$ and $k \geq 1$ cases separately.

Case 1: $k=0$. First, we have

$$
(I-B)^{\alpha} p_{0}^{\alpha, \nu}(t)=\sum_{j=0}^{\infty}(-1)^{j}\left(\begin{array}{c}
\alpha \\
j
\end{array}\right) p_{0-j}^{\alpha, \nu}(t)=p_{0}^{\alpha, \nu}(t)
$$

by (2.1); therefore,

$$
\begin{aligned}
\frac{\mathrm{d}^{\nu}}{\mathrm{d} t^{\nu}} \mathbb{P}\left(N_{\rho}^{\alpha, \nu}(t)=0\right)= & -\lambda^{\alpha}(1-\rho) p_{0}^{\alpha, \nu}(t) \\
& -\rho\left(\frac{t}{T}\right)^{\nu / \alpha}\left\{1-\mathbb{P}\left(N_{\rho}^{\alpha, \nu}(T)=0\right)\right\} t^{-\nu} \frac{\Gamma(\nu / \alpha+1)}{\Gamma(\nu / \alpha-\nu+1)}
\end{aligned}
$$

Then, by (1.2) and Remark 1.1,

$$
\begin{aligned}
\frac{\mathrm{d}^{\nu}}{\mathrm{d} t^{\nu}} \mathbb{P}\left(N_{\rho}^{\alpha, \nu}(t)=0\right)= & -\lambda^{\alpha}\left\{\mathbb{P}\left(N_{\rho}^{\alpha, \nu}(t)=0\right)\right. \\
& \left.\quad-\rho\left\{1-\left(\frac{t}{T}\right)^{\nu / \alpha}+\left(\frac{t}{T}\right)^{\nu / \alpha} \mathbb{P}\left(N_{\rho}^{\alpha, \nu}(T)=0\right)\right\}\right\} \\
& -\rho\left(\frac{t}{T}\right)^{v / \alpha}\left\{1-\mathbb{P}\left(N_{\rho}^{\alpha, \nu}(T)=0\right)\right\} t^{-v} \frac{\Gamma(\nu / \alpha+1)}{\Gamma(\nu / \alpha-v+1)}
\end{aligned}
$$

and, finally, we can check by inspection that the last equation is equivalent to that in the statement of the proposition. 
Case $2: k \geq 1$. First, again by (1.2) and Remark 1.1, we have

$$
\begin{aligned}
& \frac{\mathrm{d}^{\nu}}{\mathrm{d} t^{\nu}} \mathbb{P}\left(N_{\rho}^{\alpha, \nu}(t)=k\right) \\
& =-\lambda^{\alpha}(I-B)^{\alpha}\left[\mathbb{P}\left(N_{\rho}^{\alpha, \nu}(t)=k\right)\right. \\
& \left.\quad-\rho\left(1-\left(\frac{t}{T}\right)^{\nu / \alpha}\right) \mathbf{1}_{\{k=0\}}-\rho\left(\frac{t}{T}\right)^{\nu / \alpha} \mathbb{P}\left(N_{\rho}^{\alpha, \nu}(T)=k\right)\right] \\
& +\rho\left(\frac{t}{T}\right)^{\nu / \alpha} \mathbb{P}\left(N_{\rho}^{\alpha, \nu}(T)=k\right) t^{-\nu} \frac{\Gamma(\nu / \alpha+1)}{\Gamma(\nu / \alpha-v+1)} \\
& =-\lambda^{\alpha}(I-B)^{\alpha} \mathbb{P}\left(N_{\rho}^{\alpha, \nu}(t)=k\right)+\lambda^{\alpha} \rho\left(1-\left(\frac{t}{T}\right)^{\nu / \alpha}\right)(I-B)^{\alpha} \mathbf{1}_{\{k=0\}} \\
& +\rho\left(\frac{t}{T}\right)^{\nu / \alpha}\left[\lambda^{\alpha}(I-B)^{\alpha}+t^{-\nu} \frac{\Gamma(\nu / \alpha+1)}{\Gamma(\nu / \alpha-v+1)}\right] \mathbb{P}\left(N_{\rho}^{\alpha, \nu}(T)=k\right) .
\end{aligned}
$$

Then we obtain the desired result by noting that

$$
(I-B)^{\alpha} \mathbf{1}_{\{k=0\}}=\sum_{j=0}^{\infty}(-1)^{j}\left(\begin{array}{l}
\alpha \\
j
\end{array}\right) \mathbf{1}_{\{k-j=0\}}=(-1)^{k}\left(\begin{array}{l}
\alpha \\
k
\end{array}\right) .
$$

The proof is complete.

Finally, we remark that, even if the equations in Proposition 2.3 have some analogies with other results for fractional Poisson processes in the literature, here some standard techniques do not work because we deal with a finite-horizon time case (i.e. $t \in[0, T]$ ).

\section{A class of correlated fractional negative binomial processes}

It is well known that the negative binomial process can be seen as a suitable compound Poisson process with logarithmic distributed summands; see, e.g. [14, Proposition 1.1]. More precisely, for some $p \in(0,1)$ and some integer $r \geq 1$, we have the PGF

$$
u \mapsto h^{r}(m(u)),
$$

where $h(u):=\mathrm{e}^{\lambda(u-1)}$, with $\lambda=-\log p$, is the PGF of a Poisson distributed random variable with mean $\lambda=-\log p$, and

$$
m(u):=\frac{\log (1-(1-p) u)}{\log p} \quad \text { for }|u|<\frac{1}{1-p}
$$

is the PGF of a logarithmic distributed random variable (obviously, we have $m(u)=\infty$ if $|u| \geq 1 /(1-p))$.

In this section we present a class of correlated fractional negative binomial processes on a finite-time interval $[0, T]$. More precisely, we consider the same approach with the PGF of a space-time fractional Poisson distributed random variable; thus, for $\alpha, v \in(0,1]$, we have

$$
h_{\alpha, v}(u):=E_{v, 1}\left(-\lambda^{\alpha}(1-u)^{\alpha}\right)
$$


in place of $h$ (note that $h$ coincides with $h_{1,1}$ ), again with $\lambda=-\log p$, and this meets $g$ in (2.2) with $T=1$. Thus, we have

$$
\begin{aligned}
g(u) & :=\left\{E_{v, 1}\left(-(-\log p)^{\alpha}\left(1-\frac{\log (1-(1-p) u)}{\log p}\right)^{\alpha}\right)\right\}^{r} \\
& =\left\{E_{v, 1}\left(-\log ^{\alpha}\left(\frac{1-(1-p) u}{p}\right)\right)\right\}^{r}
\end{aligned}
$$

where, again, $r \geq 1$ is an integer power of the function $E_{v, 1}, p \in(0,1)$ and $|u|<1 /(1-p)$. We remark that $g$ in (3.1) is the PGF of $N_{\rho}(T)$, but it does not depend on $T$ as happens for $g$ in (2.2).

As far as the distribution function $F$ is concerned, we argue as in Section 2 as follows. For all $t \in[0, T]$, we want to have the condition

$$
G_{N_{0}^{\alpha, v}(t)}(u)=\left\{E_{\nu, 1}\left(-\log ^{\alpha}\left(\frac{1-(1-q(t)) u}{q(t)}\right)\right)\right\}^{r}
$$

for some $q(\cdot)$ such that $q(t) \in(0,1]$ for all $t \in[0, T]$ and $q(T)=p$. Then, by (1.1) with $\rho=0$ and by (3.1), we require that

$$
\begin{aligned}
\frac{1-(1-q(t)) u}{q(t)} & =\frac{1-(1-p)(1-F(t)+F(t) u)}{p} \\
& =\frac{1-(1-p)(1-F(t))-(1-p) F(t) u}{p}
\end{aligned}
$$

So, if we divide both numerator and denominator by $1-(1-p)(1-F(t))$, we obtain

$$
q(t)=\frac{p}{1-(1-p)(1-F(t))}
$$

Moreover, we have

$$
q(t)=\frac{p}{p+(1-p) F(t)}=\frac{1}{1+((1 / p)-1) F(t)},
$$

which yields

$$
F(t):=\frac{(1 / q(t))-1}{(1 / p)-1} \text { for } t \in[0, T],
$$

and the function $q(\cdot)$ has to be decreasing. We also give a particular example with a choice of $q(\cdot)$, and we provide the corresponding distribution function $F$.

Example 3.1. If we set

$$
q(t)=\frac{1-\lambda}{1-(1-t / T) \lambda}
$$

for some $\lambda \in(0,1)$, we recover the example in [4, Section 3.3] (see also [9, Section 4.3] for a generalization). In fact this choice of $q(\cdot)$ is the analogue of [4, Equation (3.6)]; moreover, if we set $p=1-\lambda$, we have

$$
q(t)=\frac{p}{1-(1-t / T)(1-p)}=\frac{1}{1+((1 / p)-1)(t / T)}
$$

and therefore $F(t)=t / T$. 
In what follows we present the PGFs in Proposition 3.1 and, for $r=1$ only, the corresponding PMFs in Proposition 3.2 (for $r \geq 2$ we have the $r$ th convolution of the PMF of the $r=1$ case, but we cannot provide manageable formulas). Moreover, in Proposition 3.3, we give an equation for the PGFs $\left\{G_{N_{\rho}^{\alpha, \nu}(t)}: t \in[0, T]\right\}$ in Proposition 3.1 for $r=1, \nu=\alpha$, and $\rho \in\{0,1\}$; in this case we consider fractional derivatives with respect to their argument $u$, and not with respect to time $t$.

Proposition 3.1. The PGFs $\left\{G_{N_{\rho}^{\alpha, \nu}(t)}: t \in[0, T]\right\}$ are

$$
\begin{aligned}
G_{N_{\rho}^{\alpha, v}(t)}(u)= & \rho\left(1-\frac{(1 / q(t))-1}{(1 / p)-1}\right) \\
& +\rho \frac{(1 / q(t))-1}{(1 / p)-1}\left\{E_{\nu, 1}\left(-\log ^{\alpha}\left(\frac{1-(1-p) u}{p}\right)\right)\right\}^{r} \\
& +(1-\rho)\left\{E_{\nu, 1}\left(-\log ^{\alpha}\left(\frac{1-(1-q(t)) u}{q(t)}\right)\right)\right\}^{r} .
\end{aligned}
$$

Proof. This is an immediate consequence of (1.1) and the formulas above.

In view of Proposition 3.2 some preliminaries are needed. First, we consider the Stirling numbers $\left\{s_{k, h}: k \geq h \geq 0\right\}$; see, e.g. [1, p. 824] for their definition and some properties used below. Moreover,

$$
{ }_{p} \Psi_{q}\left[\begin{array}{c}
\left(a_{1}, \alpha_{1}\right) \ldots\left(a_{p}, \alpha_{p}\right) \\
\left(b_{1}, \beta_{1}\right) \ldots\left(b_{q}, \beta_{q}\right)
\end{array}\right](z):=\sum_{j \geq 0} \frac{\prod_{h=1}^{p} \Gamma\left(a_{h}+\alpha_{h} j\right)}{\prod_{k=1}^{q} \Gamma\left(b_{k}+\beta_{k} j\right)} \frac{z^{j}}{j !}
$$

is the Fox-Wright function (see, e.g. [12, Equation (1.11.14)]) under the convergence condition

$$
\sum_{k=1}^{q} \beta_{k}-\sum_{h=1}^{p} \alpha_{h}>-1
$$

(see, e.g. [12, Equation (1.11.15)]).

Proposition 3.2. If $r=1$, the PMFs $\left\{\mathbb{P}\left(N_{\rho}^{\alpha, \nu}(t)=\cdot\right): t \in[0, T]\right\}$ are

$$
\begin{aligned}
& \mathbb{P}\left(N_{\rho}^{\alpha, \nu}(t)=k\right) \\
& \quad=(1-\rho) \mathbb{P}\left(N_{0}^{\alpha, \nu}(t)=k\right) \\
& \quad+\rho\left\{\frac{(1 / p)-(1 / q(t))}{(1 / p)-1} \mathbf{1}_{\{k=0\}}+\frac{(1 / q(t))-1}{(1 / p)-1} \mathbb{P}\left(N_{0}^{\alpha, \nu}(T)=k\right)\right\} \text { for all } k \geq 0,
\end{aligned}
$$

where, for all $t \in[0, T]$,

$$
\mathbb{P}\left(N_{0}^{\alpha, v}(t)=k\right)= \begin{cases}E_{v, 1}\left(-\log ^{\alpha}\left(1+A_{t}\right)\right) & \text { if } k=0, \\
\frac{1}{k !} \frac{\left(-A_{t}\right)^{k}}{\left(1+A_{t}\right)^{k}} \sum_{h=1}^{k} \log ^{-h}\left(1+A_{t}\right) s_{k, h} & \\
\times{ }_{2} \Psi_{2}\left[\begin{array}{cc}
(1, \alpha) & (1,1) \\
(1-h, \alpha) & (1, v)
\end{array}\right]\left(-\log ^{\alpha}\left(1+A_{t}\right)\right) & \text { if } k \geq 1,\end{cases}
$$

and $A_{t}:=(1 / q(t))-1$ (note that the convergence condition (3.3) holds because we have $\alpha+v-(\alpha+1)=v-1>-1)$. 
Proof. First, we remark that it is enough to check (3.4) (concerning the $\rho=0$ case); in fact, we obtain the formula for the general case by combining (1.2), $F$ in (3.2), and (3.4). It is well known that

$$
\mathbb{P}\left(N_{0}^{\alpha, v}(t)=k\right)= \begin{cases}G_{N_{0}^{\alpha, v}(t)}(0) & \text { if } k=0 \\ \left.\frac{1}{k !} \frac{\mathrm{d}^{k}}{\mathrm{~d} u^{k}} G_{N_{0}^{\alpha, v}(t)}(u)\right|_{u=0} & \text { if } k \geq 1\end{cases}
$$

First, if $A_{t}=(1 / q(t))-1$ as in the statement of the proposition, we have

$$
G_{N_{0}^{\alpha, \nu}(t)}(u)=E_{\nu, 1}\left(-\log ^{\alpha}\left(\frac{1-(1-q(t)) u}{q(t)}\right)\right)=E_{\nu, 1}\left(-\log ^{\alpha}\left(1+A_{t}(1-u)\right)\right)
$$

and we immediately obtain (3.4) for $k=0$. Moreover, if we prove that

$$
\begin{aligned}
& \frac{\mathrm{d}^{k}}{\mathrm{~d} u^{k}} E_{\nu, 1}\left(-\log ^{\alpha}(1+A(1-u))\right) \\
& \quad=\frac{(-A)^{k}}{(1+A(1-u))^{k}} \sum_{j \geq 0} \sum_{h=1}^{k} \frac{(-1)^{j} \Gamma(\alpha j+1)}{\Gamma(\alpha j-h+1) \Gamma(\nu j+1)} s_{k, h} \log ^{\alpha j-h}(1+A(1-u)) \\
& \quad=\frac{1}{k !} \frac{(-A)^{k}}{(1+A)^{k}} \sum_{h=1}^{k} \log ^{-h}(1+A) s_{k, h} \Psi_{2}\left[\begin{array}{rr}
(1, \alpha) & (1,1) \\
(1-h, \alpha) & (1, v)
\end{array}\right]\left(-\log ^{\alpha}(1+A)\right)
\end{aligned}
$$

for $k \geq 1$ (and for all $A \in \mathbb{R}$ ), we obtain (3.4) for $k \geq 1$ (and the proof is complete) as an immediate consequence of (3.5) and (3.7) with $A=A_{t}$. Therefore, in the remaining part of the proof we only prove (3.6) by induction; in fact, (3.7) can be checked by inspection. For $k=1$, we have

$$
\frac{\mathrm{d}}{\mathrm{d} u} E_{v, 1}\left(-\log ^{\alpha}(1+A(1-u))\right)=\sum_{j \geq 0} \frac{(-1)^{j} \alpha j}{\Gamma(\nu j+1)} \frac{\log ^{\alpha j-1}(1+A(1-u))}{1+A(1-u)}(-A),
$$

and (3.6) is proved noting that

$$
s_{1,1}=1, \quad \alpha j=\frac{\Gamma(\alpha j+1)}{\Gamma(\alpha j)} .
$$

Now we assume that (3.6) holds for $k>1$. Then, we have

$$
\begin{aligned}
\frac{\mathrm{d}^{k+1}}{\mathrm{~d} u^{k+1}} E_{v, 1}( & \left.-\log ^{\alpha}(1+A(1-u))\right) \\
=\frac{\mathrm{d}}{\mathrm{d} u}\left\{\frac{(-A)^{k}}{(1+A(1-u))^{k}}\right. & \\
& \left.\times \sum_{j \geq 0} \sum_{h=1}^{k} \frac{(-1)^{j} \Gamma(\alpha j+1)}{\Gamma(\alpha j-h+1) \Gamma(v j+1)} s_{k, h} \log ^{\alpha j-h}(1+A(1-u))\right\}
\end{aligned}
$$




$$
\begin{aligned}
=(-A)^{k}\{ & \frac{(-k)(-A)}{(1+A(1-u))^{k+1}} \\
& \times \sum_{j \geq 0} \sum_{h=1}^{k} \frac{(-1)^{j} \Gamma(\alpha j+1)}{\Gamma(\alpha j-h+1) \Gamma(v j+1)} s_{k, h} \log ^{\alpha j-h}(1+A(1-u)) \\
& +\frac{1}{(1+A(1-u))^{k}} \\
& \times \sum_{j \geq 0} \sum_{h=1}^{k} \frac{(-1)^{j} \Gamma(\alpha j+1)}{\Gamma(\alpha j-h+1) \Gamma(v j+1)} s_{k, h} \\
& \left.\times \frac{(\alpha j-h) \log ^{\alpha j-h-1}(1+A(1-u))}{1+A(1-u)}(-A)\right\}
\end{aligned}
$$

and we obtain

$$
\begin{aligned}
\frac{\mathrm{d}^{k+1}}{\mathrm{~d} u^{k+1}} E_{\nu, 1}\left(-\log ^{\alpha}(1+A(1-u))\right) \\
=\frac{(-A)^{k+1}}{(1+A(1-u))^{k+1}} \\
\quad \times\left\{-k \sum_{j \geq 0}^{k} \frac{(-1)^{j} \Gamma(\alpha j+1)}{\Gamma(\alpha j-h+1) \Gamma(\nu j+1)} s_{k, h} \log ^{\alpha j-h}(1+A(1-u))\right. \\
\left.\quad+\sum_{j \geq 0} \sum_{h=0}^{k} \frac{(-1)^{j} \Gamma(\alpha j+1)}{\Gamma(\alpha j-h) \Gamma(\nu j+1)} s_{k, h} \log ^{\alpha j-h-1}(1+A(1-u))\right\}
\end{aligned}
$$

because $(\alpha j-h) / \Gamma(\alpha j-h+1)=1 / \Gamma(\alpha j-h)$ and $s_{k, 0}=0$. Then, we obtain

$$
\begin{aligned}
\frac{\mathrm{d}^{k+1}}{\mathrm{~d} u^{k+1}} E_{v, 1}\left(-\log ^{\alpha}(1+A(1-u))\right) & \\
= & \frac{(-A)^{k+1}}{(1+A(1-u))^{k+1}} \\
& \times\left\{-k \sum_{j \geq 0} \sum_{h=1}^{k} \frac{(-1)^{j} \Gamma(\alpha j+1)}{\Gamma(\alpha j-h+1) \Gamma(v j+1)} s_{k, h} \log ^{\alpha j-h}(1+A(1-u))\right. \\
& \left.\quad+\sum_{j \geq 0} \sum_{m=1}^{k+1} \frac{(-1)^{j} \Gamma(\alpha j+1)}{\Gamma(\alpha j-m+1) \Gamma(v j+1)} s_{k, m-1} \log ^{\alpha j-(m-1)-1}(1+A(1-u))\right\} \\
& \frac{(-A)^{k+1}}{(1+A(1-u))^{k+1}} \\
& \times \sum_{j \geq 0}\left\{\sum_{h=1}^{k} \frac{(-1)^{j} \Gamma(\alpha j+1)}{\Gamma(\alpha j-h+1) \Gamma(v j+1)}\left(-k s_{k, h}+s_{k, h-1}\right) \log ^{\alpha j-h}(1+A(1-u))\right. \\
& \left.\quad+\frac{(-1)^{j} \Gamma(\alpha j+1)}{\Gamma(\alpha j-(k+1)+1) \Gamma(v j+1)} s_{k, k} \log ^{\alpha j-(k+1)}(1+A(1-u))\right\},
\end{aligned}
$$

and (3.6) holds for $k+1$ because $-k s_{k, h}+s_{k, h-1}=s_{k+1, h}$ and $s_{k, k}=s_{k+1, k+1}=1$. 
In view of Proposition 3.3 some preliminaries are needed. First, let $(O)_{\alpha}$ be the operator defined by

$$
(O)_{\alpha} f(z):= \begin{cases}\frac{1}{\Gamma(n-\alpha)} \int_{(1-a) / b}^{z} \log ^{n-1-\alpha}\left(\frac{a+b z}{a+b \tau}\right) & \\ \times\left[\left(\left(\frac{a}{b}+\tau\right) \frac{\mathrm{d}}{\mathrm{d} \tau}\right)^{n} f(\tau)\right] \frac{b}{a+b \tau} \mathrm{d} \tau & \text { if } \alpha \in(n-1, n), \\ \left(\left(\frac{a}{b}+z\right) \frac{\mathrm{d}}{\mathrm{d} z}\right)^{n} f(z) & \text { if } \alpha=n,\end{cases}
$$

where $z>(1-a) / b$. Here, for the moment, we are assuming that $\alpha>0$ and $n$ is an integer value. Thus, for $\alpha \in(n-1, n)$, this operator can be formally considered as the regularized Caputo-like fractional power of the operator $(a / b+z)(\mathrm{d} / \mathrm{d} z)$. Indeed it can be found from the definition of Caputo fractional derivative of order $\alpha$, by means of the simple transformation $z \mapsto \log (a / b+z)$. Moreover, we observe that if $a=0$ and $b=1,(3.8)$ coincides with the Caputo-like regularized Hadamard fractional derivative recently introduced in [10].

In what follows we focus our attention on the $\alpha \in(0,1)$ case and, in view of the proof of Proposition 3.3, we check that

$$
(O)_{\alpha} E_{\alpha, 1}\left(-\gamma \log ^{\alpha}(a+b z)\right)=-\gamma E_{\alpha, 1}\left(-\gamma \log ^{\alpha}(a+b z)\right) .
$$

In fact, by (3.8), for $\beta>-1$, we have

$$
\begin{aligned}
(O)_{\alpha} \log ^{\beta}(a+b z) & \\
& =\frac{1}{\Gamma(1-\alpha)} \int_{(1-a) / b}^{z} \log ^{-\alpha}\left(\frac{a+b z}{a+b \tau}\right)\left[\left(\left(\frac{a}{b}+\tau\right) \frac{\mathrm{d}}{\mathrm{d} \tau}\right) \log ^{\beta}(a+b \tau)\right] \frac{b}{a+b \tau} \mathrm{d} \tau \\
& =\frac{1}{\Gamma(1-\alpha)} \int_{(1-a) / b}^{z}(\log (a+b z)-\log (a+b \tau))^{-\alpha} \beta \frac{\log ^{\beta-1}(a+b \tau)}{a+b \tau} b \mathrm{~d} \tau
\end{aligned}
$$

and, after some computations with the change of variable $y=\log (a+b \tau) / \log (a+b z)$, we obtain

$$
(O)_{\alpha} \log ^{\beta}(a+b z)=\frac{\beta}{\Gamma(1-\alpha)} \log ^{\beta-\alpha}(a+b z) \int_{0}^{1}(1-y)^{-\alpha} y^{\beta-1} \mathrm{~d} y
$$

and, therefore,

$$
(O)_{\alpha} \log ^{\beta}(a+b z)=\frac{\Gamma(\beta+1)}{\Gamma(\beta+1-\alpha)} \log ^{\beta-\alpha}(a+b z) .
$$

Then, by (3.10) and some computations, we obtain

$$
\begin{aligned}
(O)_{\alpha} E_{\alpha, 1}\left(-\gamma \log ^{\alpha}(a+b z)\right) & =\sum_{k=1}^{\infty} \frac{(-\gamma)^{k} \log ^{\alpha k-\alpha}(a+b z)}{\Gamma(\alpha k-\alpha+1)} \\
& =-\gamma \sum_{k=0}^{\infty} \frac{(-\gamma)^{k} \log ^{\alpha k}(a+b z)}{\Gamma(\alpha k+1)}
\end{aligned}
$$

which meets (3.9). 
Proposition 3.3. Assume that $r=1$ and let $\left\{G_{N_{\rho}^{v, v}(t)}: t \in[0, T]\right\}$ be the PGFs in Proposition 3.1 with $\alpha=v$. Then we have the following results.

(i) $\left(\rho=1\right.$ case) Let $(O)_{\nu, 1}$ be the operator in (3.8) with $a=1 / p$ and $b=(p-1) / p$. Then

$$
(O)_{\nu, 1} G_{N_{1}^{v, v}(t)}(u)=-G_{N_{1}^{v, v}(t)}(u)+1-\frac{(1 / q(t))-1}{(1 / p)-1} \text { for all } u \in\left(1, \frac{1}{1-p}\right) .
$$

(ii) $(\rho=0$ case $)$ Let $(O)_{\nu, 0}$ be the operator in (3.8) with $a=1 / q(t)$ and $b=(q(t)-1) / q(t)$.

Then

$$
(O)_{\nu, 0} G_{N_{0}^{\nu, \nu}(t)}(u)=-G_{N_{0}^{\nu, \nu}(t)}(u) \text { for all } u \in\left(1, \frac{1}{1-q(t)}\right) .
$$

(iii) In both (i) and (ii), we have $G_{N_{\rho}^{\nu, v}(t)}((1-a) / b)=1$.

Proof of Proposition 3.3(i). For $\alpha=v \in(0,1)$, we have

$$
\begin{aligned}
(O)_{\nu, 1} G_{N_{1}^{\nu, \nu}(t)}(u) & =\frac{(1 / q(t))-1}{(1 / p)-1}(O)_{\nu, 1} E_{\nu, 1}\left(-\log ^{\nu}\left(\frac{1-(1-p) u}{p}\right)\right) \\
& =-\frac{(1 / q(t))-1}{(1 / p)-1} E_{\nu, 1}\left(-\log ^{\nu}\left(\frac{1-(1-p) u}{p}\right)\right) \\
& =-G_{N_{1}^{\nu, \nu}(t)}(u)+1-\frac{(1 / q(t))-1}{(1 / p)-1}
\end{aligned}
$$

where (for $\rho=1, a=1 / p, b=(p-1) / p$, and $\gamma=1$ ) we have used Proposition 3.1 and, for the second equality, (3.9). Note that we have $u \in(1,1 /(1-p))$ because $G_{N_{1}^{v, v}(t)}(u)$ is finite for $|u|<1 /(1-p)$ (see Proposition 3.1 with $\rho=1$ ) and $(1-a) / b=1$. For $\alpha=\nu=1$ it is easy to check with some computations that

$$
\left(\frac{1}{p-1}+u\right) \frac{\mathrm{d}}{\mathrm{d} u} G_{N_{1}^{1,1}(t)}(u)=-G_{N_{1}^{1,1}(t)}(u)+1-\frac{(1 / q(t))-1}{(1 / p)-1}
$$

by Proposition 3.1 (in fact we have $a / b=1 /(p-1)$ ).

Proof of Proposition 3.3(ii). For $\alpha=v \in(0,1)$, we have

$$
\begin{aligned}
(O)_{\nu, 0} G_{N_{1}^{\nu, \nu}(t)}(u) & =(O)_{\nu, 0} E_{\nu, 1}\left(-\log ^{\nu}\left(\frac{1-(1-q(t)) u}{q(t)}\right)\right) \\
& =-E_{\nu, 1}\left(-\log ^{\nu}\left(\frac{1-(1-q(t)) u}{q(t)}\right)\right) \\
& =-G_{N_{0}^{\nu, v}(t)}(u),
\end{aligned}
$$

where (for $\rho=0, a=1 / q(t), b=(q(t)-1) / q(t)$, and $\gamma=1)$ we have used Proposition 3.1 and, for the second equality, (3.9). Note that we have $u \in(1,1 /(1-p))$ arguing as we did for the proof of Proposition 3.3(i). For $\alpha=v=1$ it is easy to check with some computations that

$$
\left(\frac{1}{q(t)-1}+u\right) \frac{\mathrm{d}}{\mathrm{d} u} G_{N_{0}^{1,1}(t)}(u)=-G_{N_{0}^{1,1}(t)}(u)
$$

by Proposition 3.1 (in fact we have $a / b=1 /(q(t)-1)$ ).

Proof of Proposition 3.3(iii). The proposition trivially holds because we always have $G_{N_{\rho}^{\alpha, v}(t)}(1)=1$ (even if $\left.\alpha \neq v\right)$ and, in both Proposition 3.3(i) and (ii), $(1-a) / b=1$. 


\section{On weighted processes}

In this section we consider $\left\{N_{\rho}^{w}(t): t \in[0, T]\right\}$, where

$$
N_{\rho}^{w}(t):=\sum_{n=1}^{M_{g}^{w}} \mathbf{1}_{[0, t]}\left(X_{n}^{F, \rho}\right)
$$

and the PMF of the random variable $M_{g}^{w}$ is given by

$$
\mathbb{P}\left(M_{g}^{w}=k\right)=\frac{\mathbb{P}\left(M_{g}=k\right) w(k)}{\mathbb{E}\left[w\left(M_{g}\right)\right]} \quad \text { for all } k \geq 0
$$

for some nonnegative numbers (weights) $\{w(k): k \geq 0\}$ such that

$$
\mathbb{E}\left[w\left(M_{g}\right)\right]:=\sum_{r=0}^{\infty} w(r) \mathbb{P}\left(M_{g}=r\right) \in(0, \infty) .
$$

Then we are referring to the concept of weighted PMF; see, e.g. [11, p. 90], and the references therein.

We remark that $M_{g}^{w}$ has the same distribution of $M_{g}$ if $w(k)=1$ for all $k \geq 0$. Generally, we have the following well-known property of the weighted PMFs: if we consider 'proportional weights'

$$
\{w(k): k \geq 0\} \propto\{\tilde{w}(k): k \geq 0\},
$$

i.e. if, for some $c>0$, we have $w(k)=c \tilde{w}(k)$ for all $k \geq 0$, then we have the same weighted PMF.

The aim of this section is to illustrate the 'weighted version structure' for the PMF of $N_{\rho}^{w}(t)$ for each $t \in(0, T]$, i.e.

$$
\mathbb{P}\left(N_{\rho}^{w}(t)=k\right)=\frac{\mathbb{P}\left(N_{\rho}(t)=k\right) w(k, t)}{\mathbb{E}\left[w\left(N_{\rho}(t), t\right)\right]} \text { for all } k \geq 0
$$

for some weights $\{w(k, t): k \geq 0\}$ which depend on $t \in(0, T]$ (obviously we have $w(k, T)=$ $w(k)$ for all $k \geq 0$, i.e. (4.2) meets (4.1) when $t=T)$. Moreover, we give the corrected version of some formulas stated in [9].

Proposition 4.1. We set

$$
\begin{aligned}
q(k \mid n, F(t), \rho):= & (1-\rho)\left(\begin{array}{l}
n \\
k
\end{array}\right) F^{k}(t)(1-F(t))^{n-k} \\
& +\rho F^{k / n}(t)(1-F(t))^{1-k / n} \mathbf{1}_{\{0, n\}}(k) \text { for all } k \in\{0,1, \ldots, n\} .
\end{aligned}
$$

Then, for all $t \in(0, T]$, we have

$$
w(k, t) \propto \frac{\sum_{n=k}^{\infty} q(k \mid n, F(t), \rho) \mathbb{P}\left(M_{g}=n\right) w(n)}{\sum_{n=k}^{\infty} q(k \mid n, F(t), \rho) \mathbb{P}\left(M_{g}=n\right)} \quad \text { for all } k \geq 0 .
$$

Proof. By [9, Equation (7)], we have the following generalization of (1.3):

$$
\mathbb{P}\left(N_{\rho}(t)=k\right)=\sum_{n=k}^{\infty} q(k \mid n, F(t), \rho) \mathbb{P}\left(M_{g}=n\right) \quad \text { for all } k \geq 0 .
$$


Moreover, by (4.3) (with $N_{\rho}^{w}(t)$ and $M_{g}^{w}$ in place of $N_{\rho}(t)$ and $M_{g}$ ) and (4.1), we obtain

$$
\mathbb{P}\left(N_{\rho}^{w}(t)=k\right)=\frac{\sum_{n=k}^{\infty} q(k \mid n, F(t), \rho) \mathbb{P}\left(M_{g}=n\right) w(n)}{\mathbb{E}\left[w\left(M_{g}\right)\right]}
$$

Then (4.2) and the last equality yield

$$
\begin{aligned}
w(k, t) & =\frac{\mathbb{E}\left[w\left(N_{\rho}(t), t\right)\right]}{\mathbb{P}\left(N_{\rho}(t)=k\right)} \frac{\sum_{n=k}^{\infty} q(k \mid n, F(t), \rho) \mathbb{P}\left(M_{g}=n\right) w(n)}{\mathbb{E}\left[w\left(M_{g}\right)\right]} \\
& \propto \frac{\sum_{n=k}^{\infty} q(k \mid n, F(t), \rho) \mathbb{P}\left(M_{g}=n\right) w(n)}{\mathbb{P}\left(N_{\rho}(t)=k\right)} .
\end{aligned}
$$

We conclude the proof by taking into account (4.3) for the denominator in the last expression.

Now the correction of [9, Equations (17) and (18)]:

$$
\operatorname{cov}\left(N_{\rho}(t), N_{\rho}(s)\right)=\lambda s\{1+\lambda \rho(1-t)\}
$$

and

$$
\operatorname{cov}\left(N_{\rho}(t)-N_{\rho}(s), N_{\rho}(s)\right)=-\lambda^{2} \rho s(t-s) .
$$

We also present the corrected version of the displayed formula in [9, Example 4.1]. We refer to (1.2) in this paper and, in order to have a strict connection with the presentation in [9], we consider $t \in[0,1]$ in place of $F(t)$ with $t \in[0, T]$. We have to choose

$$
\mathbb{P}\left(N_{0}(t)=k\right)=\frac{(\lambda t)^{k}}{k !} \mathrm{e}^{-\lambda t}\left(1-t+\frac{k}{\lambda}\right) \quad \text { for all } k \geq 0
$$

for the $\rho=0$ case (see $[4$, Section 3.1]) and

$$
\mathbb{P}\left(M_{g}=k\right)= \begin{cases}\frac{\lambda^{k-1}}{(k-1) !} \mathrm{e}^{-\lambda} & \text { if } k \geq 1, \\ 0 & \text { if } k=0 .\end{cases}
$$

Then, we obtain

$$
\begin{aligned}
\mathbb{P}\left(N_{\rho}(t)=k\right)= & (1-\rho) \frac{(\lambda t)^{k}}{k !} \mathrm{e}^{-\lambda t}\left(1-t+\frac{k}{\lambda}\right) \\
& +\rho\left\{(1-t) \mathbf{1}_{\{k=0\}}+t \frac{\lambda^{k-1}}{(k-1) !} \mathrm{e}^{-\lambda} \mathbf{1}_{\{k \geq 1\}}\right\} \\
= & \begin{cases}(1-\rho) \mathrm{e}^{-\lambda t}(1-t)+\rho(1-t) & \text { if } k=0, \\
(1-\rho) \frac{(\lambda t)^{k}}{k !} \mathrm{e}^{-\lambda t}\left(1-t+\frac{k}{\lambda}\right)+\rho t \frac{\lambda^{k-1}}{(k-1) !} \mathrm{e}^{-\lambda} & \text { if } k \geq 1,\end{cases}
\end{aligned}
$$

which is the corrected version of the displayed formula in [9, Example 4.1].

\section{Acknowledgements}

We thank the referee for some useful comments and Federico Polito for Figure 1. 


\section{References}

[1] Abramowitz, M. ANd Stegun, I. A. (1964). Handbook of Mathematical Functions with Formulas, Graphs, and Mathematical Tables (National Bureau Standards Appl. Math. Ser. 55). U.S. Government Printing Office, Washington, D. C.

[2] Angulo, J. M., Ruiz-Medina, M. D., ANH, V. V. ANd GrecksCh, W. (2000). Fractional diffusion and fractional heat equation. Adv. Appl. Prob. 32, 1077-1099.

[3] Baeumer, B., Meerschaert, M. M. ANd Nane, E. (2009). Brownian subordinators and fractional Cauchy problems. Trans. Amer. Math. Soc. 361, 3915-3930.

[4] Balakrishnan, N. and Kozubowski, T. J. (2008). A class of weighted Poisson processes. Statist. Prob. Lett. 78, 2346-2352.

[5] Beghin, L. And D’Ovidio, M. (2014). Fractional Poisson process with random drift. Electron. J. Prob. 19, $26 \mathrm{pp}$.

[6] BeGHIN, L. AND MACCI, C. (2014). Fractional discrete processes: compound and mixed Poisson representations. J. Appl. Prob. 51, 19-36.

[7] Beghin, L. and Orsingher, E. (2009). Fractional Poisson processes and related planar random motions. Electron. J. Prob. 14, 1790-1827.

[8] BEghin, L. AND ORSINGHER, E. (2010). Poisson-type processes governed by fractional and higher-order recursive differential equations. Electron. J. Prob. 15, 684-709.

[9] Borges, P., Rodrigues, J. ANd Balakrishnan, N. (2012). A class of correlated weighted Poisson processes. J. Statist. Planning Inference 142, 366-375.

[10] Jarad, F., Abdeluawad, T. and Baleanu, D. (2012). Caputo-type modification of the Hadamard fractional derivatives. Adv. Difference Equations 2012, 8 pp.

[11] Johnson, N. L., Kotz, S. AND Kemp, A. W. (1992). Univariate Discrete Distributions, 2nd edn. John Wiley, New York.

[12] Kilbas, A. A., Srivastava, H. M. and Trujillo, J. J. (2006). Theory and Applications of Fractional Differential Equations. Elsevier, Amsterdam.

[13] Kostinski, A. B. (2001). On the extinction of radiation by a homogeneous but spatially correlated random medium. J. Optical Soc. Amer. A 18, 1929-1933.

[14] Kozubowski, T. J. AND PODGóRSKI, K. (2009). Distributional properties of the negative binomial Lévy process. Prob. Math. Statist. 29, 43-71.

[15] Kumar, A., Nane, E. and Vellaisamy, P. (2011). Time-changed Poisson processes. Statist. Prob. Lett. 81, 1899-1910.

[16] Laskin, N. (2003). Fractional Poisson process. Commun. Nonlinear Sci. Numer. Simul. 8, 201-213.

[17] Lefèvre, C. ANd PiCARD, P. (2011). A new look at the homogeneous risk model. Insurance Math. Econom. 49, 512-519.

[18] Mainardi, F. (1996). The fundamental solutions for the fractional diffusion-wave equation. Appl. Math. Lett. 9, 23-28.

[19] Mainardi, F., Gorenflo, R. and Scalas, E. (2004). A fractional generalization of the Poisson processes. Vietnam J. Math. 32, 53-64.

[20] Meerschaert, M. M., Nane, E. and Vellaisamy, P. (2011). The fractional Poisson process and the inverse stable subordinator. Electron. J. Prob. 16, 1600-1620.

[21] Orsingher, E. AND Beghin, L. (2004). Time-fractional telegraph equations and telegraph processes with Brownian time. Prob. Theory Relat. Fields 128, 141-160.

[22] Orsingher, E. AND BEghin, L. (2009). Fractional diffusion equations and processes with randomly-varying time. Ann. Prob. 37, 206-249.

[23] ORsingher, E. AND Polito, F. (2010). Fractional pure birth processes. Bernoulli 16, 858-881.

[24] Orsingher, E. ANd Polito, F. (2012). The space-fractional Poisson process. Statist. Prob. Lett. 82, 852-858.

[25] Podlubny, I. (1999). Fractional Differential Equations. Academic Press, San Diego, CA.

[26] Politi, M., Kaizoji, T. and Scalas, E. (2011). Full characterization of the fractional Poisson process. Europhys. Lett. 96, 20004.

[27] SchneIDER, W. R. AND WySs, W. (1989). Fractional diffusion and wave equations. J. Math. Phys. 30, $134-144$.

[28] Vellaisamy, P. and Maheshwari, A. (2014). Fractional negative binomial and Polya processes. Preprint. Available at http://arxiv.org/abs/1306.2493 